\title{
Review
}

Journal of Innate

Immunity
J Innate Immun 2009;1:88-97

DOI: 10.1159/000181181
Received: September 17, 2008

Accepted after revision: October 9, 2008 Published online: December 3, 2008

\section{Bacterial Peptidoglycan-Degrading Enzymes and Their Impact on Host Muropeptide Detection}

\author{
Jessica Humann ${ }^{a} \quad$ Laurel L. Lenz ${ }^{a, b}$ \\ a University of Colorado - Denver and ${ }^{\mathrm{b}}$ Integrated Department of Immunology, National Jewish Health, \\ Denver, Colo., USA
}

\section{Key Words}

Peptidoglycan · Autolysin · Bacteria · Subversion

\begin{abstract}
Peptidoglycan (PGN) is a major component of the bacterial cell envelope in both Gram-positive and Gram-negative bacteria. These muropeptides can be produced or modified by the activity of bacterial glycolytic and peptidolytic enzymes referred to as PGN hydrolases and autolysins. Some of these bacterial enzymes are crucial for bacterial pathogenicity and have been shown to modulate muropeptide release and/or host innate immune responses. The ability of muropeptides to modulate host responses is due to the fact that eukaryotes do not produce PGN and have instead evolved numerous strategies to detect intact PGN and PGN fragments (muropeptides). Here we review the structure of PGN and introduce the various bacterial enzymes known to degrade or modify bacterial PGN. Host factors involved in PGN and muropeptide detection are also briefly discussed, as are examples of how specific bacterial pathogens use PGN degradation and modification to subvert host innate immunity.

Copyright @ 2008 S. Karger AG, Basel
\end{abstract}

\section{Basic Peptidoglycan Structure}

The peptidoglycan (PGN) structure of both Grampositive and Gram-negative bacteria comprises repeating disaccharide backbones of $\mathrm{N}$-acetylglucosamine (NAG) and $\beta$-(1-4)-N-acetylmuramic acid (NAM) that are crosslinked by peptide stem chains attached to the NAM residues [1]. The first 2 residues of the stem peptide are generally L-alanine and D-glutamine or isoglutamine, while the last residue is typically D-alanine (fig. 1a). In contrast, the third residue of the stem peptide is a lysine in coccoid Gram-positive bacteria (such as Staphylococcus and Streptococcus species), but a meso-diaminopimelate (mDAP) residue in both Gram-negative bacteria and many rodshaped Gram-positive bacteria such as Listeria and Bacillus species [1]. In the latter (DAP-type) PGN, mDAP residues from 2 adjacent stem peptides typically link directly to each other. Conversely, the stem peptides of Lys-type PGN are bridged by a variable peptide usually comprised of 2-5 glycine and serine residues [2, 3].

\section{Common Modifications to the Basic PGN Structure}

Modifications or variations to the basic PGN structure occur frequently amongst bacterial species. Many modifications are species specific, due to the expression of unique synthetic, modifying or degradative enzymes.

Dr. Laurel L. Lenz

National Jewish Health, Integrated Department of Immunology

Rm K510, 1400 Jackson Street

Denver, CO 80206 (USA)

Tel. +1 303398 1767, Fax +1 303398 1396, E-Mail lenzl@njc.org 
However, the PGN structure of a given bacterium may also be changed in response to changes in growth conditions. The benefits of such modifications include enhanced resistance to antibiotics and host degradative enzymes that target the cell wall, and possibly impairment of detection by host innate immune receptors for intact PGN or the peptide and glycopeptide fragments of PGN commonly referred to as muropeptides.

Substitutions and modifications to the basic PGN structure occur in both the peptide stem and bridge regions and in the disaccharide backbone. The disaccharide backbone can be modified by addition of glycolic acid, via $\mathrm{N}$-acylation, to muramic acid residues - as occurs in Mycobacterium tuberculosis [4]. It is also common for bacteria to deacetylate $\mathrm{N}$-acetyl glucosamine residues [5]. In some pathogenic bacteria, such as Streptococcus pneumoniae and Listeria monocytogenes [6, 7], the deacetylases responsible for removing these acetyl groups are crucial for lysozyme resistance and virulence in infected animals. Staphylococcus aureus employs $\mathrm{O}$-acetylation as an alternative method for modification of PGN sugars to increase resistance to lysozyme [8].

Modifications to the peptide stem portion of PGN include substitutions of glycine or L-serine residues for the $\mathrm{L}$-alanine in position 1 in Mycobacterium leprae and $\mathrm{Bu}$ tyribacterium rettgeri, respectively [3]. Similarly, D-glutamine at position 2 in the stem peptide can be substituted with glycine, amidated glycine, amidated glutamine or an amidated alanine [5]. At position 3, L-ornithine, LLDAP and hydroxy-lysine can take the place of the mDAP and L-lysine [5]. Finally, the length and proportion of glycine versus serine residues in the bridge peptides of staphyloccocal Lys-type PGN can be altered by expression of Fem $A B$ and homologous nonribosomal peptidyltransferases [9-11].

Bacterial growth phase also affects PGN structure. For example, the PGN of stationary phase Bacillus subtilis is more highly cross-linked than that found in rapidly growing bacteria [12]. Likewise, Escherichia coli that is not actively growing has PGN with a higher degree of cross-linking, an increase in lipoprotein additions and shorter glycan strands compared to actively dividing E. coli [13]. Several other bacterial pathogens enter into more unique 'nongrowth states' associated with PGN modifications: Bacillus and Clostridium species substantially alter their PGN between vegetative and spore-forming stages. Pathogenic Mycobacteria do not form spores, but enter into dormant states with modified PGN structure during latent infection. When exiting nongrowth states, resuscitation-promoting factors promote cell wall

Bacterial PGN-Degrading Enzymes and Host Muropeptide Detection digestion and reactivation from dormancy in Mycobacteria $[14,15]$. Such digestion modifies the PGN structure and promotes the shift from dormancy into active growth. In addition to possible direct effects on the reactivation of latent infections, the digestion of PGN by resuscitation-promoting factors and other PGN-digesting enzymes can also lead to the release of bioactive muropeptides. The release of muropeptides from bacterial pathogens can have profound effects on pathogenesis and the initiation and regulation of host immune responses. This is because eukaryotic host organisms as diverse as legumes, insects and man have evolved multiple innate immune receptors to detect intact PGN and several distinct muropeptides, including those illustrated in figure $1 \mathrm{~b}$.

\section{Bacterial PGN Hydrolases}

Bacteria employ several classes of PGN-hydrolyzing enzymes that participate in assembly and disassembly of the bacterial cell wall during the processes of bacterial growth and division. PGN-hydrolyzing enzymes that bind to and degrade intact bacterial cells or PGN of the producing organism are classified as autolysins. Several pathogenic bacterial species have hydrolases or autolysins that are required for bacterial pathogenicity in eukaryotic hosts.

PGN hydrolases are defined by their catalytic specificities. Two classes of these enzymes function to digest the PGN glycan backbone: $\mathrm{N}$-acetylmuramidases cleave PGN between the NAG-NAM bond upstream of NAM and $\mathrm{N}$-acetylglucosaminidases cleave the NAM-NAG bond (fig. 1a). In contrast, $\mathrm{N}$-acetylmuramyl-L-alanine amidases cleave between NAM and the first alanine of the peptide chain (fig. 1a). Thus, catalysis by $\mathrm{N}$-acetylmuramyl-L-alanine amidases separate the PGN sugar backbones from the stem peptide chain $[16,17]$. Another common family of PGN hydrolase is the lytic transglycosylases (LTs). These enzymes cleave between the $\mathrm{N}$-acetylmuramic acid and $\mathrm{N}$-acetylglucosamine sugar chains (fig. 1a) to facilitate cell growth or, in some cases, to make space for secretion systems and flagella $[18,19]$ or to produce cytotoxic muropeptides [20].

Further digestion of the stem peptide requires cleavage between stem amino acids by several carboxy- and endopeptidases. Overall, a full complement of glycosidases, amidases and endopeptidases provides bacteria the capacity to fully degrade PGN and recycle cell wall glycans and amino acids. However, such complete degradation does not always occur, as several bacteria are 
a

Lys-type PGN

$1 \quad \mathrm{CH}_{2} \mathrm{OH} \quad 2 \quad \mathrm{CH}_{2} \mathrm{OH}$

$\left.\downarrow\right|_{\mathrm{H}} ^{\mathrm{OH}}$

$\stackrel{\mathrm{O}}{\mathbf{\mathrm { N }}} \mathrm{NHCOCH}_{3}$

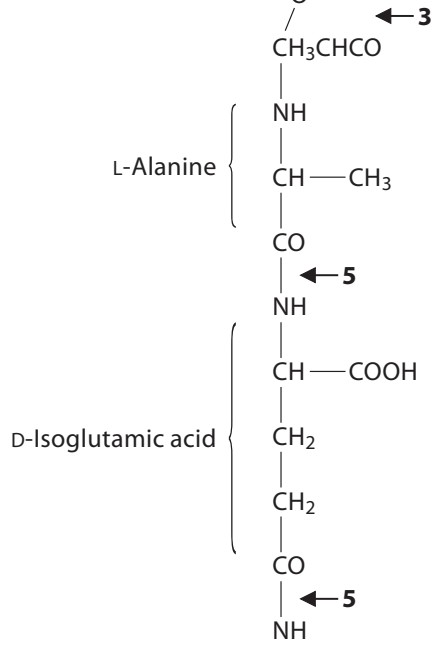

L-Lysine $\left\{\left.\right|_{\mathrm{C}} ^{\mathrm{C}} \mathrm{H}-\left(\mathrm{CH}_{2}\right)_{4}-\mathrm{CH}_{2}-\mathrm{NH}_{2}\right.$<smiles>[CH]NC(=O)[O-]</smiles>

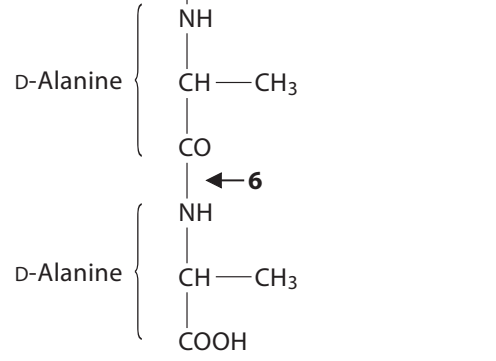

b $\quad T C T$

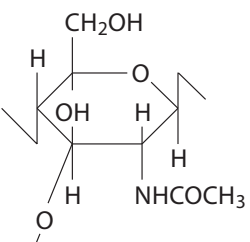

$\mathrm{CH}_{3} \mathrm{CHCO}$

L-Ala

Glu

mDAP

D-Ala

(G)MDP
DAP-type PGN
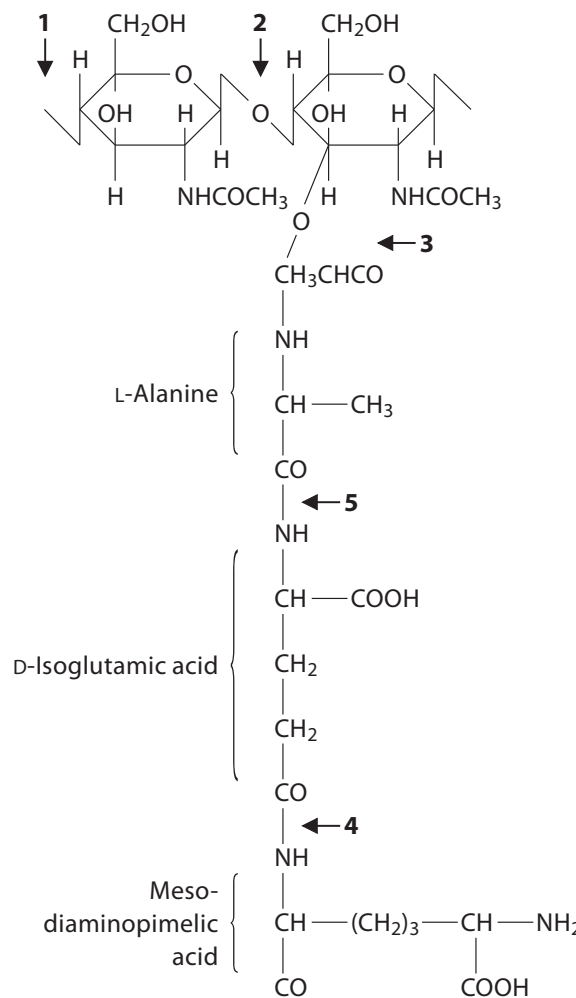

D-Alanine \{

$\mathrm{NH}$

$\mathrm{CH}-\mathrm{CH}_{3}$

$\mathrm{CO}$

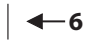

D-Alanine $\left\{\begin{array}{l}\mathrm{CH} \\ \left.\right|_{\mathrm{COOH}} ^{\mathrm{H}}-\mathrm{CH}_{3}\end{array}\right.$

(G)MTP

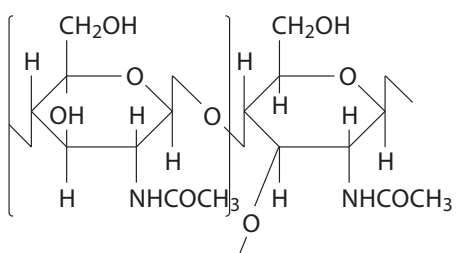

$\mathrm{CH}_{3} \mathrm{CHCO}$

L-Ala

Glu

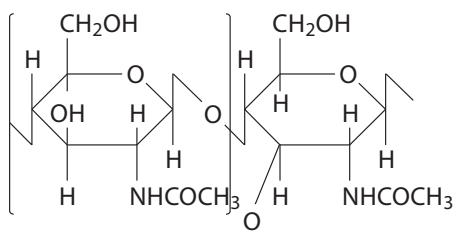

O

$\mathrm{CH}_{3} \mathrm{CHCO}$

L-Ala

Glu

mDAP 
known to release cell wall fragments (muropeptides) that exert potent biological effects on the host [21]. Such bioactive muropeptides include a disaccharide-tetrapeptide fragment of PGN known as tracheal cytotoxin (TCT; fig. 1b). TCT is released by both Bordatella pertussis and Neisseria gonorrhoeae and causes death of tracheal and vaginal ciliated epithelial cells as well as induction of slow-wave sleep [22-26]. Muramyldipeptide (MDP) and related muropeptides (for example, GMDP) influence the activation of host immune responses and have adjuvant activity in promoting antibody responses (fig 1b).

\section{Domain Structures of PGN Hydrolases}

Sequence motifs indicative of conserved catalytic and noncatalytic domains are both typically found in the primary amino acid sequences of PGN hydrolases. Thus, bioinformatics analyses can be used to estimate the distribution of hydrolases with a given predicted catalytic specificity within the genomes of different pathogen species and strains. Such analyses reveal that there are often multiple enzymes with the same predicted cleavage specificity within a given organism. Furthermore, the numbers of enzymes with a particular specificity varies between species or strains. For instance, Bacillus anthracis Sterne encodes 9 genes with type 2 or $3 \mathrm{~N}$-acetylmuramoyl-L-alanine amidase domains (EC:3.5.1.28). In contrast, M. tuberculosis H37Rv has only 4 and L. EGD-e has only 3 genes with such domains (fig. 2a). The selective pressure for retaining such redundancy is not clear. However, variations in the assortments of putative noncatalytic do-

Fig. 1. Structure of Lys-type and DAP-type PGN. a Lys-type PGN contains a lysine at the third position of the peptide stem and is predominantly seen in Gram-positive bacterial species. DAPtype PGN contains a mDAP at the third position of the peptide stem and is predominantly seen in Gram-negative bacterial species. Arrows indicate sites of PGN cleavage by PGN hydrolytic enzymes: $1=\mathrm{N}$-acetylglucosaminidases; $2=\mathrm{N}$-acetylmuramidases; 3 = N-acetylmuramyl-L-alanine amidases; 4 = glutamyl-mesodiaminopimelate endopeptidases of the p60/NLP family; 5 = other endopeptidases; 6 = carboxy- and trans-peptidases such as those of the penicillin-binding proteins (PBP) family. Lytic transglycosylases can also cleave between the sugar backbone moieties (1 and 2). b Biologically active PGN fragments (muropeptides) include tracheal cytotoxin (TCT), (glutamyl)muramyldipeptide [(G)MDP] and (glutamyl)-muramyltetrapeptide [(G)MTP]. (G)MDP triggers host Nod2 and NALP3 activation, while murine Nod 1 detects both TCT and MTP.

Bacterial PGN-Degrading Enzymes and Host Muropeptide Detection mains, and in some case the absence of obvious secretion signal peptides, suggest that these enzymes differ in their regulation and/or localization within or outside the bacterium. Given that the expression patterns of these genes may also vary, it is possible that individual enzymes with apparently identical catalytic specificities may play distinct roles in the biology or pathogenicity of a given bacterium. Indeed, the greater number of $\mathrm{N}$-acetylmuramyl$\mathrm{L}$-alanine amidases found in the genome of $B$. anthracis Sterne may reflect the need for unique or 'extra' amidase activity during sporulation or spore germination.

Redundancy is also seen in another class of PGN hydrolases, D-glutamate-mDAP endopeptidases, which cleave between the third and fourth amino acid residues of the stem peptide in DAP-type PGN. D-glutamate-mDAP endopeptidase activity is associated with the so-called NlpC/ P60 domain based on experimental evidence from studies with the B. subtilis LytF protein [27]. NlpC/P60 domains are typically found at the $\mathrm{C}$-terminus and contain a conserved C-terminal histidine residue. Multiple proteins with NlpC/P60 domains are found in individual Grampositive bacteria. Deletion of the B. subtilis lytF gene impaired bacterial cell wall separation and caused filamentous growth (chaining) of bacteria [27]. Another B. subtilis protein containing an NlpC/P60 domain is CwlS, which cleaves PGN at the D-g-glutamyl-meso-diaminopimelate bond and is localized to the cell poles/separation sites of the bacteria [28]. These data suggest possible roles for CwlF and CwlS in B. subtilis septation or other processes that occur at these sites. L. monocytogenes codes for at least 3 proteins with NlpC/P60 domains: p60, p45 and Lmo0394 (fig. 2b). The C-terminus of p45 is highly homologous to the same region in p60 [29]. However, based on the inability to engineer deletion mutants of $\mathrm{p} 45$, this enzyme appears to be essential for viability of $L$. monocytogenes. Conversely, neither p60 nor Lmo0394 are required for L. monocytogenes viability [30].

Other conserved domains associated with PGN hydrolytic activity include the cysteine- and histidine-dependent amidohydrolase/peptidase (CHAP) domain [31, 32], which was first identified in the S. aureus LytA autolysin but is also present in E. coli glutathionylspermidine synthetase, the putative lysin protein from Streptococcus pyogenes and lytic enzymes from Streptococcus agalactiae bacteriophage $\mathrm{B} 30$ [33] and $S$. aureus phage phi MR11 [34]. Interestingly, the CHAP domain is often found in association with other putative catalytic PGN hydrolase domains within a single protein. Thus, CHAP domains may enable a single enzyme to have multiple or novel catalytic specificities.

J Innate Immun 2009;1:88-97 
Fig. 2. Pathogenic bacteria encode variable repertoires of 'redundant' PGN-hydrolyzing enzymes that may permit unique biological functions. a Considerable variation is seen in the numbers of PGN-hydrolyzing amidases and domain assortments within these enzymes between $B$. anthracis Sterne, M. tuberculosis $\mathrm{H} 37 \mathrm{Rv}$ and $L$. monocytogenes EGDe. Shown are SLH domains, LGFP domains and PG-binding-1 domains, all of which are distinct putative PGN cell wall-binding domains. Variability in these domains may facilitate distinct binding or cleavage specificities for the various amidases, perhaps to target specific PGN substructures or muropeptides. Protein names listed are NCBI designations. b Domain structures of the $3 \mathrm{~L}$. monocytogenes PGN hydrolases that contain a NlpC/p60 catalytic domain. SH3 is a proline-binding domain and LysM is a PGN-binding domain, while functions of the threonine-asparagine repeat region are unknown. These related enzymes appear to have distinct functions, since mutation of p60 in L. monocytogenes $10403 \mathrm{~S}$ appears to uniquely reduce pathogenicity and host NK cell responses in infected animals.

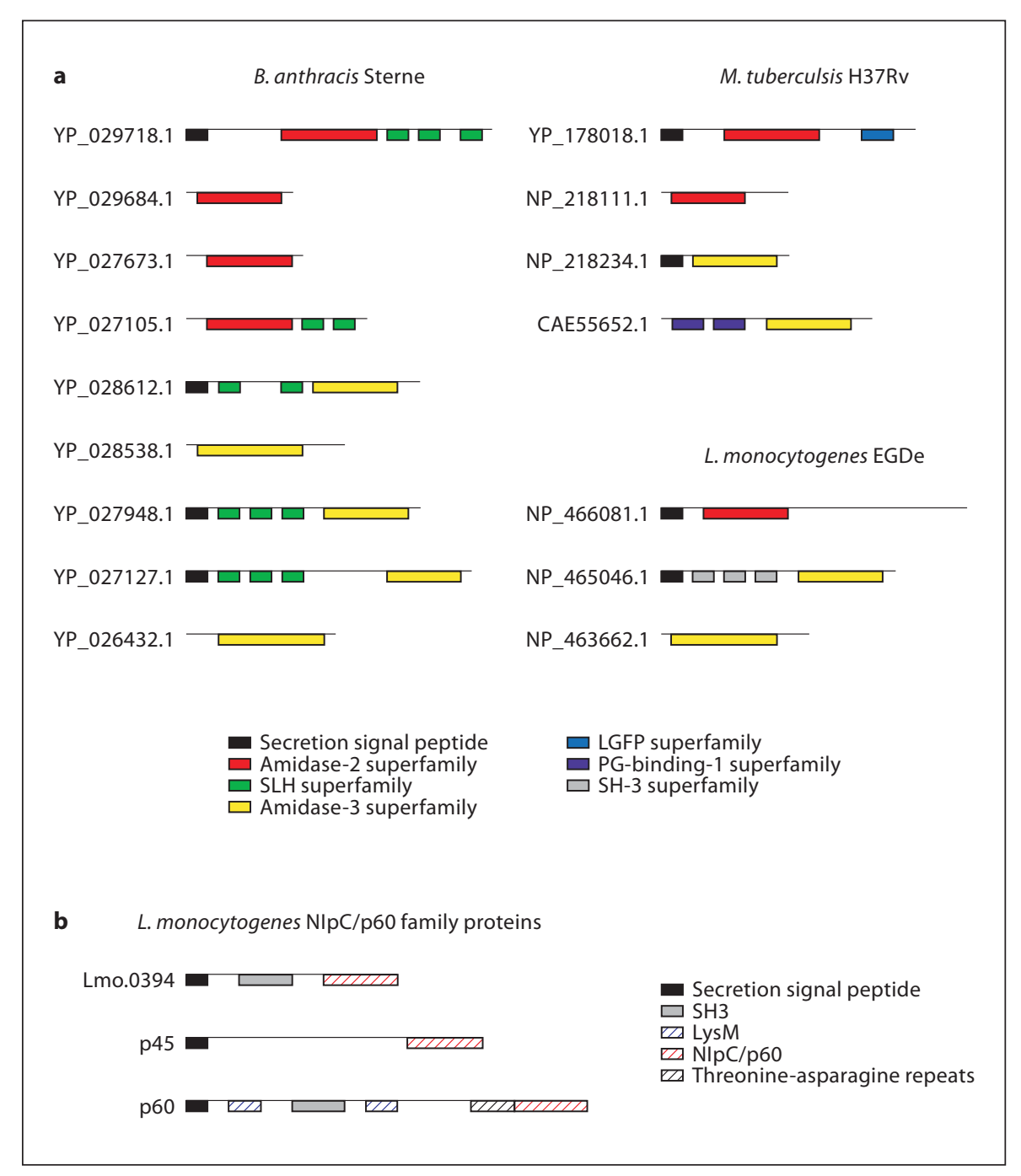

PGN hydrolases and modifying enzymes also typically contain one or more conserved domains not associated with catalytic activity per se. Such noncatalytic domains may assist in localizing these enzymes to specific sites on the bacterial cell wall or permit their association with bacterial cofactors or perhaps host proteins. Examples of noncatalytic domains commonly found in bacterial PGN hydrolases include the GW domains that are associated with binding to lipoteichoic acid, SH3-like domains that may interact with carbohydrates and/or polyproline stretches, FtsN PGN-binding domains, choline-binding domains and LysM domains [35]. Interestingly, LysMlike domains are also found in receptor kinases important for recognition of bacterial nodulation factors during the establishment of symbiotic rhizobial infections in legumous plants [36, 37].

\section{Host Detection of PGN and Muropeptides}

The ability of PGN and muropeptides to influence the biology of eukaryotes results from the triggering of eukaryotic receptor systems that have evolved to detect these molecules. On the eukaryotic host cell surface, Tolllike receptor (TLR) 2 recognizes PGN or molecules associated with PGN $[38,39]$. PGN or muropeptides also bind cell surface and soluble peptidoglycan recognition proteins (PGRPs). The 17 Drosophila PGRPs positively or negatively influence the activation of host innate immune responses to bacteria [40-43]. Mammals also produce 4 known PGRPs: PGRP-S, PGRP-I $\alpha$, PGRP-I $\beta$ and PGRP-L [44]. However, unlike insect PGRPs, mammalian orthologs have no known signaling capabilities. Rather, some human PGRPs bind directly to the PGN on 
invading pathogens and are thus thought to interfere with PGN synthesis [45]. PGRP-L also has $\mathrm{N}$-acetylmuramoyl-L-alanine amidase activity [46], and thus digests PGN at position 3 in figure 1a. This enzymatic activity may digest intact bacteria and/or reduce host pro-inflammatory responses to PGN or muropeptides [47]. However, while PGRP-L contributes to antibacterial Drosophila immune responses [48-50], its influence on mammalian innate immunity is less clear [51].

Certain members of the Nod-like receptor (NLR) family also detect specific muropeptides. NLR proteins include NALPs, NODs and NAIPs, and are generally expressed constitutively by antigen-presenting cells and epithelia. They are also often induced by inflammation and inflammatory/immune regulatory cytokines such as TNF- $\alpha$ and IFN- $\gamma$ [52]. The C-terminal leucine-rich repeat domains of NLRs are related to the extracellular (ligand-binding) regions of TLRs. Certain NLRs also contain a NACHT or NOD domain for oligomerization, as well as 1 or more protein-protein interaction domains at their N-termini $[52,53]$. Signaling through NLR proteins profoundly influences the regulation of host immune responses. For instance, mutations in the nod 2 gene are risk factors for the development of Crohn's disease [54-56], an inflammatory disorder of the intestines. Nod 2 contributes to host recognition of MDP and regulates the activity of host NF- $\kappa \mathrm{B}$ family transcription factors $[57,58]$. Human Nod1 also influences NF- $\mathrm{BB}$ activity, but senses distinct, mDAP-containing muropeptides derived from DAP-type PGN. Nod1 detects MTP and related structures (fig. 1b). The minimum structure sensed by Nod1 is a dipeptide comprised of L-glutamate-mDAP [59]. Like Nod2, the NALP3 NLR protein also appears to be activated by MDP $[60,61]$. NALP3 forms a complex with Caspase 1 and other components to form an inflammasome that regulates the activation and secretion of pro-IL-1b and other substrates. Also like nod2, mutations in nod1 and nalp 3 genes predispose to inflammatory diseases, including asthma and Muckle-Wells syndrome $[62,63]$.

\section{Subversion of Muropeptide Detection and Immune Responses}

The activity of PGN hydrolases and autolysins can promote release of various muropeptides into an infected host or host cell or may, in some cases, destroy the ability of specific muropeptides to trigger PGRPs or NLRs. Hence, PGN hydrolytic activity and other mechanisms that skew muropeptide release can modify the host re- sponse in a manner favorable for the pathogen. Indeed, evidence from studies with several bacterial pathogens support the concept that PGN digestion and modification are common pathogenic strategies to subvert muropeptide recognition and host innate immunity.

\section{N. gonorrhoeae}

AmiC is an N. gonorrhoeae N-acetylmuramyl-L-alanine amidase active during cell separation. Deletion of this autolysin results in growth inhibition and also reduced PGN fragment release. Specifically, metabolic labeling studies revealed that $\mathrm{AmiC}$ mutants fail to release PGN disaccharides [64]. Thus, AmiC may help reduce release of specific immune-stimulatory muropeptides. The putative $N$. gonorrhoeae LTs, LtgE and LtgD, also degrade PGN into monomers and free disaccharides. $N$. gonorrhoeae double mutants lacking both of these LTs released no PGN monomers or free disaccharide from the bacterial cell wall [20]. LtgE and LtgD are thought to degrade larger PGN fragments into smaller forms, as a way to break down the cell wall to components available for recycling into new PGN. Such activities may enable the bacterium to evade specific host muropeptide detection systems. In contrast, the N. gonorrhoeae LT LtgA appears to specifically enhance the production of PGN cytotoxin. This cytotoxic fragment of PGN is identical to the bioactive TCT muropeptide released from B. pertussis [65]. Bacteria deficient in LtgA show a decrease in PGN turnover during log phase growth, as well as decreases in PGN monomer and free disaccharide release. Thus, the major function of LtgA may be to induce the killing of ciliated cells via PGN cytotoxin/TCT release, which may in turn prohibit ciliary expulsion of invading bacteria. Enhancement of the inflammatory response and influx of inflammatory cells such as neutrophils are a by-product of the PGN release and may also contribute to dissemination of N. gonorrhoeae [20].

In addition to autolysins, several transporters and recycling machinery are required for efficient PGN turnover and muropeptide release. In N. gonorrhoeae, AmpG is a cytoplasmic PGN transporter and AmpD is an amidase that is specific for anhydro-muropeptides, which it separates into stem peptides and sugar residues. AmpD deficiency inhibits breakdown of larger PGN fragments and AmpG mutants release more PGN monomers [66]. Thus, changes in expression of AmpG and AmpD may further modulate release of TCT and other muropeptides. 


\section{B. pertussis}

B. pertussis TCT is a DAP-containing muropeptide, GlcNAc-(anhydro)MurNAc-L-Ala-D-Glu-mesoDAP-DAla, present in DAP-type PGN and released during log phase growth. In humans, PGRPI $\alpha \mathrm{C}$ binds to TCT, and in Drosophila TCT binds PGRP-LC and activates the Imd pathway. The result of TCT recognition is the destruction of lung and vaginal ciliated epithelial cells - as seen in whopping cough and gonorrhea [67]. TCT also induces production of TNF- $\alpha$, IL- 6 , IL- $1 \beta$ and KC secretion in wild-type mouse peritoneal macrophages, but not Nod1deficient macrophages. The synthetic agonist FK156 also induces cytokine responses via Nod1, and can synergize with endotoxin to induce nitric oxide and cause epithelial damage.

In addition to releasing toxic PGN fragments, autolysins in $B$. pertussis also modify PGN to allow the release of other virulence factors into host cells. PtlE is a PGN hydrolase that has an active site similar to glycohydrolases [68]. PtlE deletion mutants or mutants with PtlE protein that lacks catalytic activity are both deficient in secretion of pertussis toxin, an ADP ribosyltransferase that interferes with signaling through $G$ protein-coupled receptors. Thus, PGN hydrolysis by PtlE results helps $B$. pertussis to impair migration of host inflammatory cells.

\section{Helicobacter pylori}

H. pylori is a Gram-negative bacterial pathogen that infects the gastric mucosa. In the course of infection, H. pylori transfers PGN fragments directly into gastric epithelial cells via a type IV secretion system [69]. Bacterial mutants that lack the cagPAI genes that encode for this secretion system fail to induce NF- $\kappa B$ signaling via Nod1. The Nod1 specificity of this interaction was also shown using dominant-negative CARD domain Nod1 mutants and siRNA to Nod1. Cells expressing such mutant Nod1 forms showed reduced activation of NF- $\kappa \mathrm{B}$ and production of IL- 8 during $H$. pylori infection [69]. The LT activity of Slt enzymes appears to produce the muropeptides secreted by the cag secretion system [70]. Similarly, Shigella flexneri triggers Nod1-dependent responses and expresses a homolog of Slt, known as SltY.

$H$. pylori also undergoes shape changes during different phases of growth. In vitro, the bacterium is a curved rod, whereas the mature infectious form of the bacterium assumes a coccoid morphology. These coccoid $H$. pylori are abundant in severely damaged regions of infected stomachs [71]. The PGN of both curved and coccoid forms of $H$. pylori is rich in pentapeptide side chain muropeptides. However, in the coccoid form there is an accumulation of dipeptide monomers and a decrease in tri- and tetrapeptide fragments. During stationary phase there is also an increase in the number of glycine-containing peptides in H. pylori PGN [71]. Since epithelial cells require Nod1 signaling to induce inflammatory responses, the morphological changes in $H$. pylori appear to help it dampen an inflammatory immune responses and escape detection [72]. Thus, PGN from the spiral form of $H$. pylori activates NF- $\kappa \mathrm{B}$ via Nod1 and leads to IL- 8 production, but the coccoid form activates NF- $\kappa B$ via Nod2 and fails to induce IL-8. AmiA is a PGN hydrolase important in the change from spiral to coccoid forms [72]. AmiA deletion mutants of $H$. pylori are impaired in their ability to change into coccoids and deficient in the release of the Nod2 agonist GMDP. These data suggest a contribution of increased Nod2 signaling to reduced host inflammatory responses against $H$. $p y$ lori. Furthermore, defective Nod 2 signaling may predispose to inflammation-induced cancer, since the Nod2 R702W mutation has been associated with gastric lymphoma [73].

\section{M. tuberculosis}

Like H. pylori, M. tuberculosis employs autolysins that alter the PGN layer between different growth states. Instead of changing shapes, $M$. tuberculosis moves from active to dormant growth states. The resuscitation-promoting factors (Rpfs) are PGN-hydrolyzing enzymes involved in this growth state shift from dormancy into reactivation of the bacterium. Some of these Rpf proteins, specifically RpfB and RpfE, associate with RipA and colocalize to the septae of the cells [14]. M. tuberculosis deficient in 4 (ABCD) or even 5 (ABCDE) of the 5 Rpfs show delayed colony formation on agar plates, but are viable. However, such strains are avirulent in mice [14]. Conversely, deletion of any single Rpf is not sufficient to cause virulence or reactivation defects. Thus, some of the Rpfs may be able to compensate for the loss of others. However, deletion of both RpfA and RpfB in M. tuberculosis results in a defect in reactivation from dormancy [15]. Furthermore, bone marrow-derived macrophages infected with RpfA and RpfB double mutants produced approximately 2 -fold higher levels of IL- 6 and TNF- $\alpha$ compared to wild-type M. tuberculosis infection [15]. Thus, Rpf proteins and their PGN products may also reduce pro-inflammatory responses during reactivation of $M$. tuberculosis from dormancy. 


\section{L. monocytogenes}

L. monocytogenes uses both autolysins and deacetylases to influence PGN structure and release as well as host immune responses. The autolysins p60 and NamA are secreted by the SecA2 secretion system and cleave PGN at sites predicted to increase release of the Nod2 agonist muropeptide, GMDP [70]. The p60 autolysin may also alone disrupt Nod1-dependent responses by cleaving the D-glutamate-mDAP bond. Work from our laboratory has also shown that the p60 autolysin is required for L. monocytogenes virulence and also that expression of p60 enhances NK cell activation during the innate immune response to this pathogen [74]. Depletion of NK cells reduces L. monocytogenes growth in infected animals $[75,76]$, thus p60dependent enhancement of the NK cell response may improve bacterial growth and dissemination within the host.

The cell surface autolysin IspC [77] has also been implicated in L. monocytogenes virulence [78]. While IspC deletion does not impact in vitro growth of the bacteria, it does show decreased bacterial burden and mortality in infected mice. Like the PtlE hydrolase in B. pertussis, IspC has function beyond PGN hydrolysis, as it appears to be required for surface display of other virulence factors, such as the L. monocytogenes ActA protein [78]. Finally, NAG fragments of PGN may be deacetylated into glucosamine by the L. monocytogenes $\mathrm{N}$-deacetylase. Deletion of this gene renders L. monocytogenes more susceptible to lysozyme and impairs its access to the host cell cytosol [7]. Fully acylated PGN fragments are more readily detected by Nod proteins, so the deacylation of L. monocytogenes PGN may allow the pathogen to avoid signaling via Nod proteins during infection.

\section{Conclusions}

Recent years have seen the identification and study of several host pathways that detect intact bacterial PGN or muropeptides, including TLRs, PGRPs and NLR family proteins. Conservation of such pathways in mice, man and other eukaryotes highlights their importance but also provides selective pressure for bacterial virulence mechanisms that subvert PGN and muropeptide detection. Bacteria employ multiple enzymes that regulate PGN modifications and release of muropeptides. In some cases these enzymes promote generation of 'stealth' muropeptides that are not detected by the host, as seen with the Rpf and AmiA enzymes of the chronic bacterial pathogens M. tuberculosis and H. pylori. For other bacteria, including B. pertussis, $N$. gonorrhoeae, S. pneumoniae and L. monocytogenes, pathogenesis requires specific PGN hydrolases or other enzymes that permit release of bioactive muropeptides like TCT, (G)MTP, and (G)MDP. Although detection of (G)MPD by Nod2 appears to prevent chronic inflammation and tissue damage associated with Crohn's disease, the detection of such muropeptides by most PGRPs, Nod1 or NALP3 generally increases inflammation. It thus remains to be determined how the sum of the various host muropeptide detection systems influences immune activation and the context in which such activation can promote bacterial pathogenicity. Future studies in the field should more precisely delineate how bacterial PGN degradation and modification machinery influence host inflammatory and antibacterial immune responses. A better understanding of the effects of muropeptide detection should ultimately improve therapeutic strategies for directing host inflammatory responses in the context of colonization by commensal versus pathogenic bacteria.

\section{References}

$\checkmark 1$ Bourhis LL, Werts C: Role of Nods in bacterial infection. Microbes Infect 2007;9:629636.

2 Guan R, Roychowdhury A, Ember B, Kumar S, Boons GJ, Mariuzza RA: Structural basis for peptidoglycan binding by peptidoglycan recognition proteins. Proc Natl Acad Sci USA 2004; 101:17168-17173.

-3 Vollmer W, Blanot D, de Pedro MA: Peptidoglycan structure and architecture. FEMS Microbiol Rev 2008;32:149-167.

4 Brennan PJ: Structure, function, and biogenesis of the cell wall of Mycobacterium tuberculosis. Tuberculosis (Edinb) 2003;83:91-97.

Bacterial PGN-Degrading Enzymes and Host Muropeptide Detection
${ }_{5}$ Traub S, von Aulock S, Hartung T, Hermann C: MDP and other muropeptides - direct and synergistic effects on the immune system. J Endotoxin Res 2006;12:69-85.

-6 Vollmer W, Tomasz A: Peptidoglycan Nacetylglucosamine deacetylase, a putative virulence factor in Streptococcus pneumoniae. Infect Immun 2002;70:7176-7178.

7 Boneca IG, Dussurget O, Cabanes D, Nahori MA, Sousa S, Lecuit M, Psylinakis E, Bouriotis V, Hugot JP, Giovannini M, Coyle A, Bertin J, Namane A, Rousselle JC, Cayet N, Prevost MC, Balloy V, Chignard M, Philpott DJ, Cossart P, Girardin SE: A critical role for peptidoglycan N-deacetylation in Listeria evasion from the host innate immune system. Proc Natl Acad Sci USA 2007;104:997-1002.

-8 Bera A, Biswas R, Herbert S, Kulauzovic E, Weidenmaier C, Peschel A, Gotz F: Influence of wall teichoic acid on lysozyme resistance in Staphylococcus aureus. J Bacteriol 2007; 189:280-283.

9 Ehlert K, Schroder W, Labischinski H: Specificities of FemA and FemB for different glycine residues: FemB cannot substitute for FemA in staphylococcal peptidoglycan pentaglycine side chain formation. J Bacteriol 1997;179:7573-7576. 
-10 Hegde SS, Shrader TE: FemABX family members are novel nonribosomal peptidyltransferases and important pathogen-specific drug targets. J Biol Chem 2001;276: 6998-7003.

- 11 Sugai M, Fujiwara T, Ohta K, Komatsuzawa $\mathrm{H}$, Ohara M, Suginaka H: epr, which encodes glycylglycine endopeptidase resistance, is homologous to $f e m A B$ and affects serine content of peptidoglycan cross bridges in Staphylococcus capitis and Staphylococcus aureus. J Bacteriol 1997; 179:4311-4318.

-12 Atrih A, Bacher G, Allmaier G, Williamson MP, Foster SJ: Analysis of peptidoglycan structure from vegetative cells of Bacillus subtilis 168 and role of PBP 5 in peptidoglycan maturation. J Bacteriol 1999;181:39563966.

$\checkmark 13$ Keep NH, Ward JM, Cohen-Gonsaud M, Henderson B: Wake up! Peptidoglycan lysis and bacterial non-growth states. Trends $\mathrm{Mi}$ crobiol 2006;14:271-276.

- 14 Kana BD, Gordhan BG, Downing KJ, Sung N, Vostroktunova G, Machowski EE, Tsenova L, Young M, Kaprelyants A, Kaplan G, Mizrahi V: The resuscitation-promoting factors of Mycobacterium tuberculosis are required for virulence and resuscitation from dormancy but are collectively dispensable for growth in vitro. Mol Microbiol 2008;67: 672-684.

15 Russell-Goldman E, Xu J, Wang X, Chan J, Tufariello JM: A Mycobacterium tuberculosis Rpf double-knockout strain exhibits profound defects in reactivation from chronic tuberculosis and innate immunity phenotypes. Infect Immun 2008;76:4269-4281.

- 16 Fournier B, Philpott DJ: Recognition of Staphylococcus aureus by the innate immune system. Clin Microbiol Rev 2005;18:521540.

17 Scheurwater EM, Pfeffer JM, Clarke AJ: Production and purification of the bacterial autolysin $\mathrm{N}$-acetylmuramoyl-L-alanine amidase B from Pseudomonas aeruginosa. Protein Expr Purif 2007;56:128-137.

$\checkmark 18$ Scheurwater E, Reid CW, Clarke AJ: Lytic transglycosylases: bacterial space-making autolysins. Int J Biochem Cell Biol 2008;40: 586-591.

19 Zahrl D, Wagner M, Bischof K, Bayer M, Zavecz B, Beranek A, Ruckenstuhl C, Zarfel GE, Koraimann G: Peptidoglycan degradation by specialized lytic transglycosylases associated with type III and type IV secretion systems. Microbiology 2005;151:3455-3467.

20 Cloud-Hansen KA, Hackett KT, Garcia DL, Dillard JP: Neisseria gonorrhoeae uses two lytic transglycosylases to produce cytotoxic peptidoglycan monomers. J Bacteriol 2008; 190:5989-5994.

- 21 Park JT, Uehara T: How bacteria consume their own exoskeletons (turnover and recycling of cell wall peptidoglycan). Microbiol Mol Biol Rev 2008;72:211-227.
22 Cookson BT, Cho HL, Herwaldt LA, Goldman WE: Biological activities and chemical composition of purified tracheal cytotoxin of Bordetella pertussis. Infect Immun 1989; 57:2223-2229.

23 Cookson BT, Tyler AN, Goldman WE: Primary structure of the peptidoglycan-derived tracheal cytotoxin of Bordetella pertussis. Biochemistry 1989;28:1744-1749.

24 Martin SA, Karnovsky ML, Krueger JM, Pappenheimer JR, Biemann K: Peptidoglycans as promoters of slow-wave sleep. I. Structure of the sleep-promoting factor isolated from human urine. J Biol Chem 1984; 259:12652-12658.

25 Melly MA, McGee ZA, Rosenthal RS: Ability of monomeric peptidoglycan fragments from Neisseria gonorrhoeae to damage human fallopian-tube mucosa. J Infect Dis 1984;149:378-386.

26 Sinha RK, Rosenthal RS: Release of soluble peptidoglycan from growing conococci: demonstration of anhydro-muramyl-containing fragments. Infect Immun 1980;29:914-925.

27 Anantharaman V, Aravind L: Evolutionary history, structural features and biochemical diversity of the NlpC/P60 superfamily of enzymes. Genome Biol 2003;4:R11.

28 Fukushima T, Afkham A, Kurosawa S, Tanabe T, Yamamoto H, Sekiguchi J: A new D,L-endopeptidase gene product, YojL (renamed CwlS), plays a role in cell separation with LytE and LytF in Bacillus subtilis. J Bacteriol 2006; 188:5541-5550.

29 Schubert K, Bichlmaier A, Mager E, Wolff K, Ruhland G, Fiedler F: P45, an extracellular $45 \mathrm{kDa}$ protein of Listeria monocytogenes with similarity to protein p60 and exhibiting peptidoglycan lytic activity. Arch Microbiol 2000;173:21-28.

30 Lenz LL, Mohammadi S, Geissler A, Portnoy DA: SecA2-dependent secretion of autolytic enzymes promotes Listeria monocytogenes pathogenesis. Proc Natl Acad Sci USA 2003; 100:12432-12437.

31 Bateman A, Rawlings ND: The CHAP domain: a large family of amidases including GSP amidase and peptidoglycan hydrolases. Trends Biochem Sci 2003;28:234-237.

- 32 Layec S, Decaris B, Leblond-Bourget N: Characterization of proteins belonging to the CHAP-related superfamily within the Firmicutes. J Mol Microbiol Biotechnol 2008; 14:31-40.

33 Donovan DM, Foster-Frey J, Dong S, Rousseau GM, Moineau S, Pritchard DG: The cell lysis activity of the Streptococcus agalactiae bacteriophage B30 endolysin relies on the cysteine, histidine-dependent amidohydrolase/peptidase domain. Appl Environ Microbiol 2006;72:5108-5112.

34 Rashel M, Uchiyama J, Takemura I, Hoshiba H, Ujihara T, Takatsuji H, Honke K, Matsuzaki S: Tail-associated structural protein gp61 of Staphylococcus aureus phage phi MR11 has bifunctional lytic activity. FEMS Microbiol Lett 2008;284:9-16.
35 Vollmer W, Joris B, Charlier P, Foster S: Bacterial peptidoglycan (murein) hydrolases. FEMS Microbiol Rev 2008;32:259-286.

36 Limpens E, Franken C, Smit P, Willemse J, Bisseling T, Geurts R: LysM domain receptor kinases regulating rhizobial Nod factor-induced infection. Science 2003;302:630-633.

- 37 Madsen EB, Madsen LH, Radutoiu S, Olbryt M, Rakwalska M, Szczyglowski K, Sato S, Kaneko T, Tabata S, Sandal N, Stougaard J: A receptor kinase gene of the LysM type is involved in legume perception of rhizobial signals. Nature 2003;425:637-640.

- 38 Travassos LH, Girardin SE, Philpott DJ, Blanot D, Nahori MA, Werts C, Boneca IG: Tolllike receptor 2-dependent bacterial sensing does not occur via peptidoglycan recognition. EMBO Rep 2004;5:1000-1006.

39 Dziarski R, Gupta D: Staphylococcus aureus peptidoglycan is a toll-like receptor 2 activator: a reevaluation. Infect Immun 2005;73: 5212-5216.

40 Dziarski R: Peptidoglycan recognition proteins (PGRPs). Mol Immunol 2004;40:877886

41 Gupta D: Peptidoglycan recognition proteins-maintaining immune homeostasis and normal development. Cell Host Microbe 2008;3:273-274.

42 Lhocine N, Ribeiro PS, Buchon N, Wepf A, Wilson R, Tenev T, Lemaitre B, Gstaiger M, Meier P, Leulier F: PIMS modulates immune tolerance by negatively regulating Drosophila innate immune signaling. Cell Host Microbe 2008;4:147-158.

43 Maillet F, Bischoff V, Vignal C, Hoffmann J, Royet J: The Drosophila peptidoglycan recognition protein PGRP-LF blocks PGRP-LC and IMD/JNK pathway activation. Cell Host Microbe 2008;3:293-303.

44 McDonald C, Inohara N, Nunez G: Peptidoglycan signaling in innate immunity and inflammatory disease. J Biol Chem 2005;280: 20177-20180.

45 Cho S, Wang Q, Swaminathan CP, Hesek D, Lee M, Boons GJ, Mobashery S, Mariuzza RA: Structural insights into the bactericidal mechanism of human peptidoglycan recognition proteins. Proc Natl Acad Sci USA 2007;104:8761-8766.

46 Wang ZM, Li X, Cocklin RR, Wang M, Fukase K, Inamura S, Kusumoto S, Gupta D, Dziarski R: Human peptidoglycan recognition protein- $\mathrm{L}$ is an $\mathrm{N}$-acetylmuramoyl-Lalanine amidase. J Biol Chem 2003;278: 49044-49052.

47 Hoijer MA, Melief MJ, Debets R, Hazenberg MP: Inflammatory properties of peptidoglycan are decreased after degradation by human $\mathrm{N}$-acetylmuramyl-L-alanine amidase. Eur Cytokine Netw 1997;8:375-381.

48 Choe KM, Werner T, Stoven S, Hultmark D, Anderson KV: Requirement for a peptidoglycan recognition protein (PGRP) in Relish activation and antibacterial immune responses in Drosophila. Science 2002;296: 359-362. 
-49 Gottar M, Gobert V, Michel T, Belvin M, Duyk G, Hoffmann JA, Ferrandon D, Royet J: The Drosophila immune response against Gram-negative bacteria is mediated by a peptidoglycan recognition protein. Nature 2002;416:640-644.

50 Ramet M, Manfruelli P, Pearson A, MatheyPrevot B, Ezekowitz RA: Functional genomic analysis of phagocytosis and identification of a Drosophila receptor for E. coli. Nature 2002;416:644-648.

$51 \mathrm{Xu} \mathrm{M}$, Wang Z, Locksley RM: Innate immune responses in peptidoglycan recognition protein L-deficient mice. Mol Cell Biol 2004;24:7949-7957.

-52 Chamaillard M, Girardin SE, Viala J, Philpott DJ: Nods, Nalps and Naip: intracellular regulators of bacterial-induced inflammation. Cell Microbiol 2003;5:581-592.

-53 Sirard JC, Vignal C, Dessein R, Chamaillard M: Nod-like receptors: cytosolic watchdogs for immunity against pathogens. PLoS Pathog 2007;3:e152.

54 Hampe J, Cuthbert A, Croucher PJ, Mirza MM, Mascheretti S, Fisher S, Frenzel H, King K, Hasselmeyer A, MacPherson AJ, Bridger S, van Deventer S, Forbes A, Nikolaus S, Lennard-Jones JE, Foelsch UR, Krawczak M, Lewis C, Schreiber S, Mathew CG: Association between insertion mutation in NOD2 gene and Crohn's disease in German and British populations. Lancet 2001;357: 1925-1928.

-55 Hugot JP, Chamaillard M, Zouali H, Lesage S, Cezard JP, Belaiche J, Almer S, Tysk C, O'Morain CA, Gassull M, Binder V, Finkel Y, Cortot A, Modigliani R, Laurent-Puig P, Gower-Rousseau C, Macry J, Colombel JF, Sahbatou M, Thomas G: Association of NOD2 leucine-rich repeat variants with susceptibility to Crohn's disease. Nature 2001; 411:599-603.

- 56 Ogura Y, Bonen DK, Inohara N, Nicolae DL, Chen FF, Ramos R, Britton H, Moran T, Karaliuskas R, Duerr RH, Achkar JP, Brant SR, Bayless TM, Kirschner BS, Hanauer SB, Nunez G, Cho JH: A frameshift mutation in NOD2 associated with susceptibility to Crohn's disease. Nature 2001;411:603-606.

-57 Girardin SE, Boneca IG, Viala J, Chamaillard M, Labigne A, Thomas G, Philpott DJ, Sansonetti PJ: Nod2 is a general sensor of peptidoglycan through muramyl dipeptide (MDP) detection. J Biol Chem 2003;278:8869-8872.

58 Inohara N, Ogura Y, Fontalba A, Gutierrez O, Pons F, Crespo J, Fukase K, Inamura S, Kusumoto S, Hashimoto M, Foster SJ, Moran AP, Fernandez-Luna JL, Nunez G: Host recognition of bacterial muramyl dipeptide mediated through NOD2. Implications for Crohn's disease. J Biol Chem 2003;278:5509-5512.
9 Girardin SE, Boneca IG, Carneiro LA, Antignac A, Jehanno M, Viala J, Tedin K, Taha MK, Labigne A, Zahringer U, Coyle AJ, DiStefano PS, Bertin J, Sansonetti PJ, Philpott DJ: Nod 1 detects a unique muropeptide from Gram-negative bacterial peptidoglycan. Science 2003;300:1584-1587.

60 Martinon F, Agostini L, Meylan E, Tschopp J: Identification of bacterial muramyl dipeptide as activator of the NALP3/cryopyrin inflammasome. Curr Biol 2004;14:19291934.

61 Pan Q, Mathison J, Fearns C, Kravchenko VV, Da Silva Correia J, Hoffman HM, Kobayashi KS, Bertin J, Grant EP, Coyle AJ, Sutterwala FS, Ogura Y, Flavell RA, Ulevitch RJ: MDP-induced interleukin- $1 \beta$ processing requires Nod2 and CIAS1/NALP3. J Leukoc Biol 2007;82:177-183.

62 Hoffman HM, Mueller JL, Broide DH, Wanderer AA, Kolodner RD: Mutation of a new gene encoding a putative pyrin-like protein causes familial cold autoinflammatory syndrome and Muckle-Wells syndrome. Nat Genet 2001;29:301-305.

63 Hysi P, Kabesch M, Moffatt MF, Schedel M, Carr D, Zhang Y, Boardman B, von Mutius E, Weiland SK, Leupold W, Fritzsch C, Klopp N, Musk AW, James A, Nunez G, Inohara N, Cookson WO: NOD1 variation, immunoglobulin $\mathrm{E}$ and asthma. Hum Mol Genet 2005;14:935-941.

64 Garcia DL, Dillard JP: AmiC functions as an $\mathrm{N}$-acetylmuramyl-L-alanine amidase necessary for cell separation and can promote autolysis in Neisseria gonorrhoeae. J Bacteriol 2006;188:7211-7221.

65 Cloud KA, Dillard JP: A lytic transglycosylase of Neisseria gonorrhoeae is involved in peptidoglycan-derived cytotoxin production. Infect Immun 2002;70:2752-2757.

66 Garcia DL, Dillard JP: Mutations in ampG or ampD affect peptidoglycan fragment release from Neisseriagonorrhoeae. J Bacteriol 2008; 190:3799-3807.

67 Swaminathan CP, Brown PH, Roychowdhury A, Wang Q, Guan R, Silverman N, Goldman WE, Boons GJ, Mariuzza RA: Dual strategies for peptidoglycan discrimination by peptidoglycan recognition proteins (PGRPs). Proc Natl Acad Sci USA 2006;103: 684-689.

68 Rambow-Larsen AA, Weiss AA: The PtlE protein of Bordetella pertussis has peptidoglycanase activity required for Ptl-mediated pertussis toxin secretion. J Bacteriol 2002; 184:2863-2869.
69 Viala J, Chaput C, Boneca IG, Cardona A, Girardin SE, Moran AP, Athman R, Memet S, Huerre MR, Coyle AJ, DiStefano PS, Sansonetti PJ, Labigne A, Bertin J, Philpott DJ, Ferrero RL: Nod1 responds to peptidoglycan delivered by the Helicobacter pylori cag pathogenicity island. Nat Immunol 2004;5: 1166-1174.

70 Boneca IG: The role of peptidoglycan in pathogenesis. Curr Opin Microbiol 2005;8: 46-53.

71 Costa K, Bacher G, Allmaier G, DominguezBello MG, Engstrand L, Falk P, de Pedro MA, Garcia-del Portillo F: The morphological transition of Helicobacter pylori cells from spiral to coccoid is preceded by a substantial modification of the cell wall. J Bacteriol 1999;181:3710-3715.

72 Chaput C, Ecobichon C, Cayet N, Girardin SE, Werts C, Guadagnini S, Prevost MC, Mengin-Lecreulx D, Labigne A, Boneca IG: Role of AmiA in the morphological transition of Helicobacter pylori and in immune escape. PLoS Pathog 2006;2:e97.

-73 Rosenstiel P, Hellmig S, Hampe J, Ott S, Till A, Fischbach W, Sahly H, Lucius R, Folsch UR, Philpott D, Schreiber S: Influence of polymorphisms in the NOD1/CARD4 and NOD2/CARD15 genes on the clinical outcome of Helicobacter pylori infection. Cell Microbiol 2006;8:1188-1198.

74 Humann J, Bjordahl R, Andreasen K, Lenz LL: Expression of the p 60 autolysin enhances NK cell activation and is required for Listeria monocytogenes expansion in IFN- $\gamma$ responsive mice. J Immunol 2007; 178:24072414.

75 Schultheis RJ, Kearns RJ: In vivo administration of anti-asialo-GM1 antibody enhances splenic clearance of Listeria monocytogenes. Nat Immun Cell Growth Regul 1990;9:376386.

76 Teixeira HC, Kaufmann SH: Role of NK1.1+ cells in experimental listeriosis: $\mathrm{NK} 1+$ cells are early IFN- $\gamma$ producers but impair resistance to Listeria monocytogenes infection. J Immunol 1994;152:1873-1882.

77 Wang L, Lin M: Identification of IspC, an 86kilodalton protein target of humoral immune response to infection with Listeria monocytogenes serotype $4 \mathrm{~b}$, as a novel surface autolysin. J Bacteriol 2007;189:20462054.

78 Wang L, Lin M: A novel cell wall-anchored peptidoglycan hydrolase (autolysin), IspC, essential for Listeria monocytogenes virulence: genetic and proteomic analysis. $\mathrm{Mi}$ crobiology 2008;154:1900-1913. 